\title{
Structure Loaded Vacuum Laser-Driven Particle Acceleration Experiments at SLAC
}

\author{
Tomas Plettner ${ }^{1}$, Robert L. Byer ${ }^{1}$, Eric R. Colby ${ }^{2}$, Benjamin M. Cowan ${ }^{2}$, \\ Rasmus Ischebeck ${ }^{2}$, Christopher McGuinness ${ }^{2}$, Melissa R. Lincoln ${ }^{2}$, \\ Christopher M.S. Sears ${ }^{2}$, Robert H. Siemann ${ }^{2}$, James E. Spencer ${ }^{2}$ \\ 1 - Stanford University, Stanford, CA 94305 \\ 2 - Stanford Linear Accelerator Center, Menlo Park, CA 94025
}

\begin{abstract}
We present an overview of the future laser-driven particle acceleration experiments. These will be carried out at the E163 facility at SLAC. Our objectives include a reconfirmation of the proof-of-principle experiment, a staged buncher laser-accelerator experiment, and longerterm future experiments that employ dielectric laser-accelerator microstructures.
\end{abstract}

PACS: 41.75.Jv, 41.75.Ht, 42.25.Bs

\section{INTRODUCTION}

The near-future experiments described here build on the results of the initial proofof-principle demonstration for laser-driven particle acceleration from a boundaryterminated vacuum [1]. The first set of experiments will explore the concept of laserdriven particle acceleration as an inverse transition radiation process (ITR) under various geometrical configurations and different boundary conditions. Upon concluding these tests, our focus will shift to a two-stage buncher-accelerator experiment. It will bear some resemblance to the STELLA experiment carried out with two IFELs at 10 micron radiation [2], but will differ in that the second accelerator is not an IFEL and that the wavelength will be an order of magnitude shorter, at the red edge of the visible spectrum $(\lambda=800 \mathrm{~nm})$. Finally, dielectric microstructures of various geometries will be explored as laser-driven particle accelerators. These include hollow core photonic bandgap fiber accelerators as proposed by X.E. Lin [3] and 3dimensional photonic bandgap structures such as the "woodpile" lattice explored by B. Cowan [4].

This proceeding article focuses on the proposed ITR experiments. We summarize the important results of the initial proof-of-principle demonstration and follow with an in-depth description of the follow-up experiments that will employ the singleboundary geometry. We finalize the article with an overview of the longer-term twostage and photonic bandgap accelerator experiments. 


\section{KEY RESULTS FROM THE PROOF-OF-PRINCIPLE EXPERIMENT}

The initial proof-of-principle experiment for laser-driven particle acceleration in vacuum employed the simplest conceivable geometry to demonstrate this particle acceleration mechanism. A single linearly polarized laser beam was oriented at a shallow angle with respect to the electron beam trajectory and interacted with the relativistic electrons in the upstream space of a thin reflective boundary, in a similar fashion as proposed by Edinghofer and Pantell in 1979 [5].

The source of the relativistic electrons for proof-of-principle experiment was the SCA-FEL facility at Stanford University. The SCA produced a $30 \mathrm{MeV}$ electron beam with an ultra-low energy spread necessary for this type of single-interaction experiment. Table 1 summarizes the important laser and electron beam parameters.

TABLE 1. Laser and electron beam parameters of the LEAP experiment

\begin{tabular}{|c|c|}
\hline \multicolumn{2}{|c|}{ Laser beam parameters } \\
\hline Wavelength $\lambda$ & $800 \mathrm{~nm}$ \\
\hline Waist FWHM spot size & $110 \mu \mathrm{m}$ \\
\hline FWHM pulse duration & 2-4 psec \\
\hline Crossing angle $\alpha$ & 3-20 mrad \\
\hline Laser pulse energy & $1 / 2 \mathrm{~mJ} /$ pulse \\
\hline Laser repetition rate & $1 \mathrm{kHz}$ \\
\hline \multicolumn{2}{|c|}{ Electron beam parameters } \\
\hline Beam energy & $30 \mathrm{MeV}$ \\
\hline Macro pulse repetition rate & $10 \mathrm{~Hz}$ \\
\hline Micro pulse repetition rate & $11.7 \mathrm{MHz}$ \\
\hline FWHM spot size at the focus & $50 \mu \mathrm{m}$ \\
\hline FWHM pulse duration & $1-2$ psec \\
\hline Initial energy spread & $25-30 \mathrm{keV}$ \\
\hline Charge/bunch at the experiment & $\sim 1 \mathrm{pC}$ \\
\hline
\end{tabular}

A high resolution $90^{\circ}$ bending magnet located downstream of the laser-electron interaction region was employed to observe the energy spectrum of the electron beam. Since the electron beam was not optically bunched the electron beam was spread over all possible laser optical phases and therefore the laser-driven particle acceleration effect manifested itself as an increase of the energy spread of the electron beam in the presence of the laser beam.

Both the laser and the electron beam had a 1-2 psec pulse structure. The data was taken in the form of laser time scans in the vicinity of the temporal-overlap condition with the electron bunch. Due to the natural shot-to-shot jitter of the energy spread of the electron beam it proved convenient to randomly toggle the laser as the data was being collected and to generate separate laser-on and a laser-off data sets. The key results of the data scans are listed below 


\section{Verification of the Lawson-Woodward Theorem}

To verify the Lawson-Woodward Theorem two successive laser time scans, one with the boundary in place and the following with the tape moved out were taken. Figure 1 shows two successive laser time scans; Figure 1a corresponds to data taken with the boundary in place and Figure $1 \mathrm{~b}$ with the boundary removed. As observed in Figure 1a there is a clear laser-driven energy modulation that peaks at a laser timing of 295 psec. In Figure 1b it can be observed that the laser induced energy modulation disappears when the boundary is removed

a)

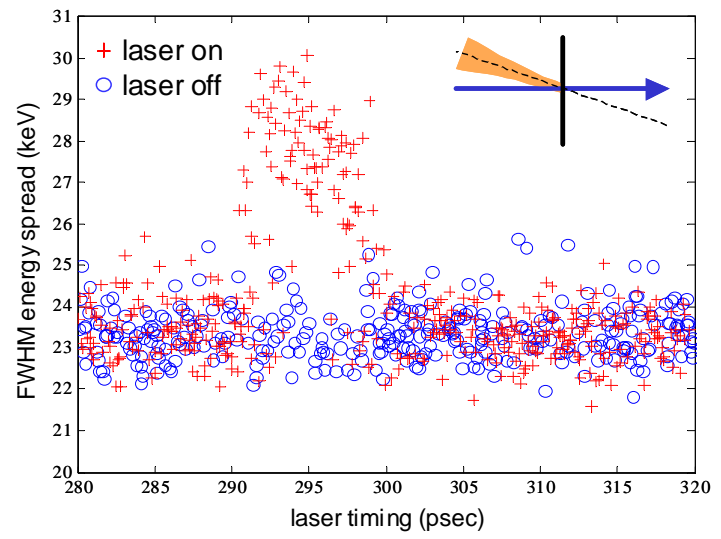

b)

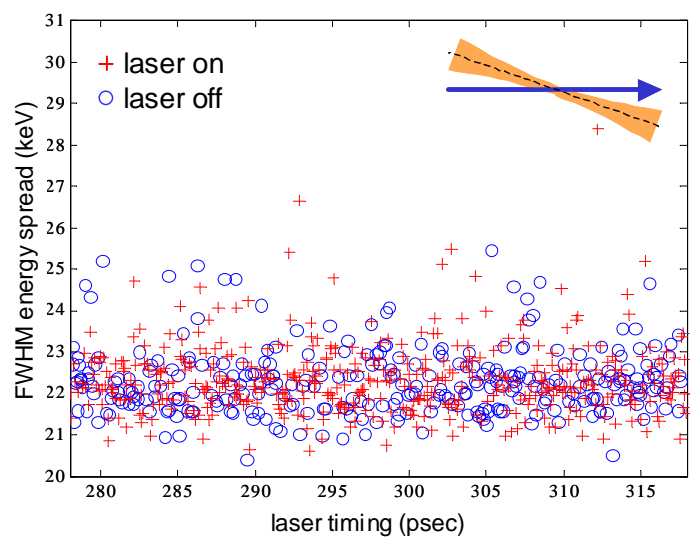

FIGURE 1. The effect of the field-terminating boundary

\section{Dependence on the laser polarization and the laser electric field amplitude}

Figure 2a shows the observed average energy modulation as a function laser polarization, which is found to be in good agreement with the expected cosine dependence. Figure $2 \mathrm{~b}$ shows the observed dependence of on the peak electric field of the laser. The average energy modulation varies linearly with the incident laser electric field as expected from theory and shows no significant offset from the origin.

These measurements constitute the experimental observation of laser-driven acceleration of relativistic electrons in vacuum by a properly terminated, linearly polarized visible laser beam. The energy modulation was confirmed to scale linearly with the longitudinal component of the electric field of the laser, to follow a cosine dependence on the polarization, and to require a boundary to limit spatial the interaction of the incident laser beam with the electron beam. 
a) Laser Polarization Angle Dependence

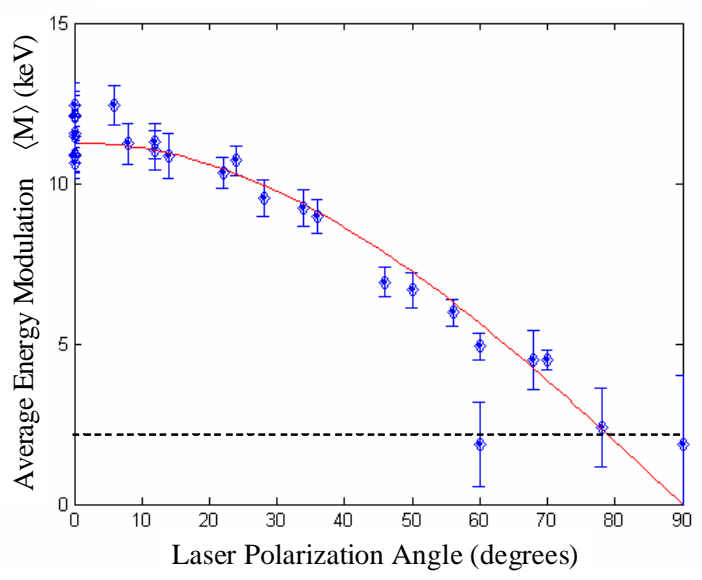

b) Laser Electric Field Dependence

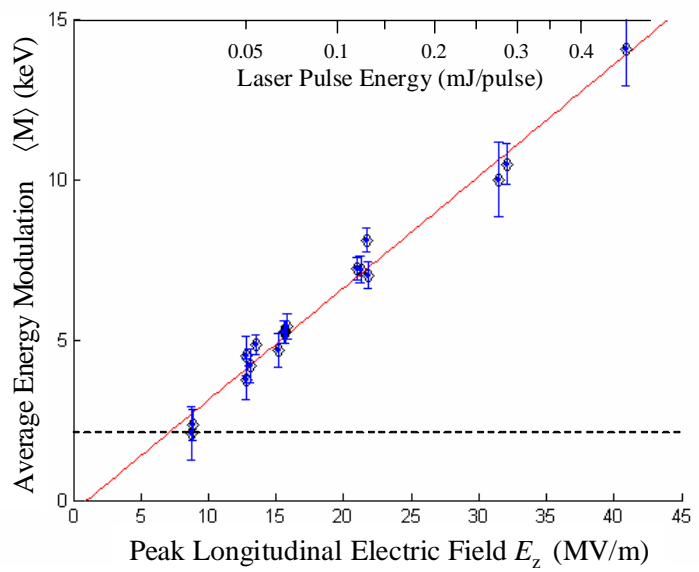

FIGURE 2. a) Dependence of the average energy modulation on the laser polarization angle. The red curve is a cosine function fit to the data. B) Dependence of the average energy modulation on the laser peak electric field. The red line is a linear fit to the data. The dashed lines in both plots show the noise floor limit.

\section{THE INVERSE TRANSITION RADIATION EXPERIMENTS}

The inverse-radiation picture is a powerful theorem applicable to many particle acceleration mechanisms. In this picture the energy gain of the charged particle from the external electromagnetic field is described as the overlap integral of the particle's wake fields and the driving electromagnetic wave that are radiated to infinity. It is assumed that only the charged particle can exchange net energy with the field. Furthermore the energy that is naturally lost from the particle's wake-field radiation in the presence of the structure is assumed to be negligible compared to the energy gain or loss from the interaction with the external field. With these assumptions it has been shown that the energy gain of the charged particle can be described by the formula $[6,7]$.

is the vacuum impedance, and and are the phasors from the laser component leaving the volume of interest and the wake-filed respectively. Equation 1 shows that in the inverse-radiation picture the particle's energy gain scales as the total overlap between the driving field that leaves the volume and the radiated wake field.

The proof-of-principle setup with a single semi-infinite boundary and a single laser beam is an ideal geometry for testing the equivalence relation of equation 1 . For such 
a setup we compare the electron energy gain calculated by the path integral of the electric field observed by the particle (LHS of equation 1) to the overlap integral of the laser filed pattern radiated to infinity with the transition radiation pattern of the electron from the boundary (RHS of equation 1). Of interest to us are three different types of boundaries:

- Reflective

- Absorptive

- Transparent

Figure 3 illustrates these three cases. Case (a) was already partially carried out for proof-of-principle demonstration. In the upcoming experiments we will complement this case by measuring the laser crossing angle dependence and exploring the condition for oblique boundaries.

(a)

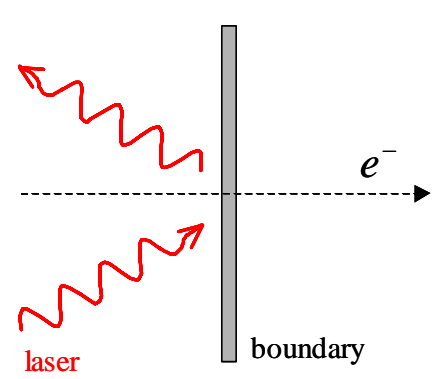

(b)

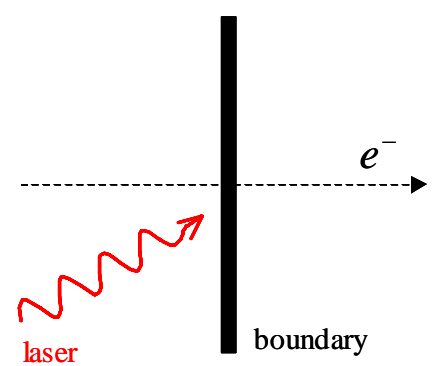

(c)

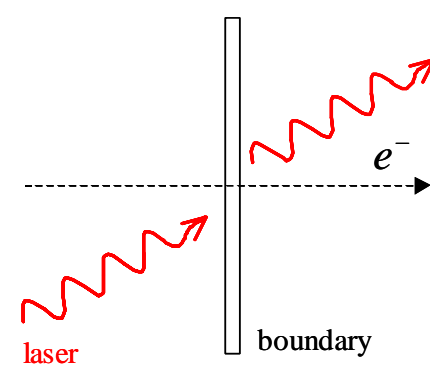

FIGURE 3. Laser acceleration from a (a) reflective boundary, (b) absorptive "black" boundary, and (c) a lossless transparent boundary

\section{Case (a): The reflective boundary}

We will allow the reflective planar boundary to be oriented at arbitrary obliquity angles with respect to the electron beam and will scan the laser-crossing angle with respect to the electron beam. The laser-electron interaction will occur in the upstream region of the boundary, as illustrated in Figure 4. The electron beam travels in the zdirection and the orientation of the boundary is allowed to be at an arbitrary angle $\xi / 2$ with respect to the electron beam. The laser beam is assumed to be a linearly polarized monochromatic plane wave at an angle $\alpha$ with respect to the electron beam. We idealize the boundary as a perfect conductor and therefore the radiative wake-field of the electron corresponds to the well-known transition radiation field distribution from the flat infinite reflective surface, and can be evaluated, for example, by the method of image charges [9] or by a plane wave decomposition approach [10]. 


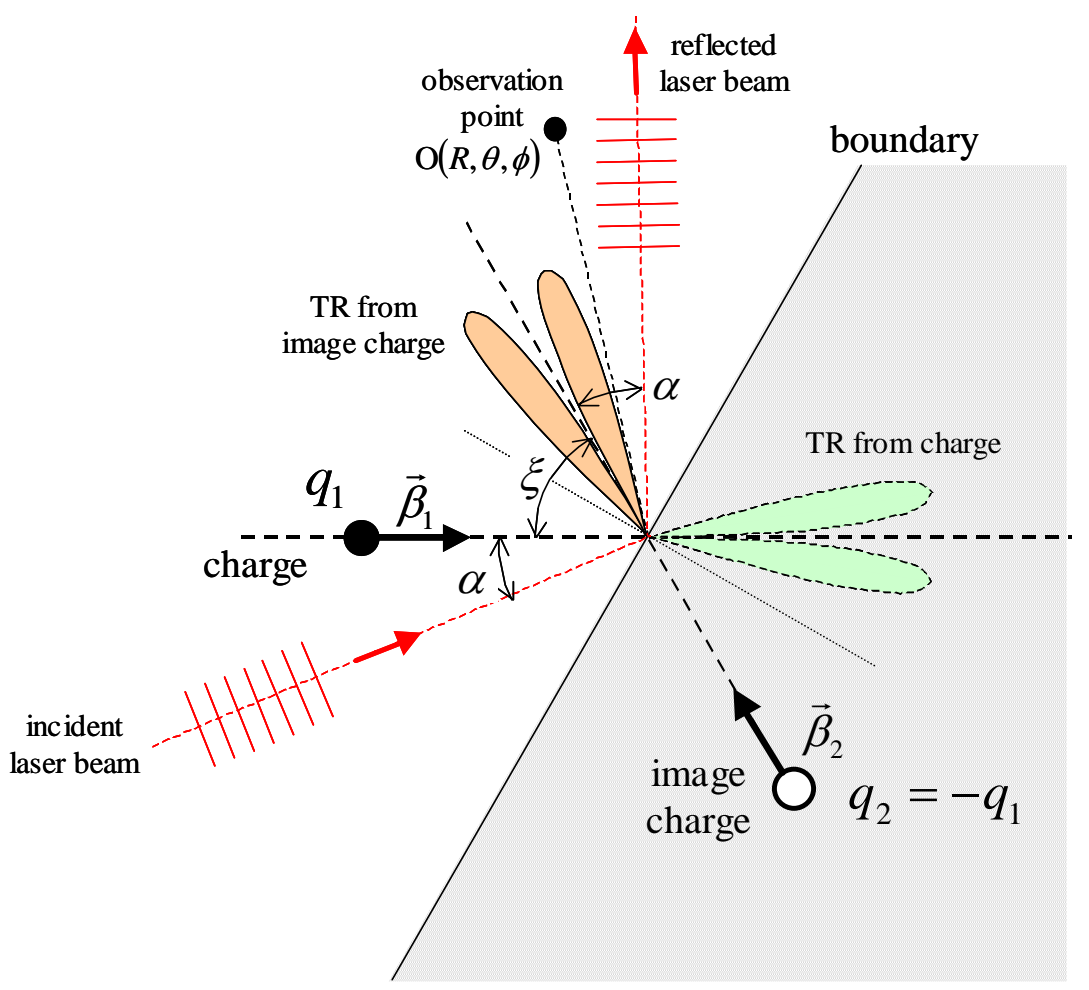

FIGURE 4. Representation of the particle and fields with a reflective boundary at an arbitrary orientation to the particle's trajectory

As can be seen in Figure 4 the transition radiation cone of the image charge shows the largest overlap with the reflected laser beam and therefore has the largest contribution to the overlap integral with the reflected laser beam. Evaluating the overlap of the transition radiation pattern and the reflected laser field in the relativistic limit where $\gamma>>1$ yields and expression for the electron energy gain that takes the simple form

where $q$ is the electron charge, the electric field amplitude, , $\lambda$ the wavelength, the laser polarization angle, the optical phase, and the laser crossing angle. Equation 2 shows that the electron energy gain is expected to be proportional to and , to depend on the laser polarization angle and on the optical phase of the laser, and to show the expected dependence on the laser-crossing angle that follows the transition radiation cone amplitude and has a maximum at . The path integral method yields the same prediction for the energy gain displayed in equation 2. Although equation 2 was derived for the relativistic limit the equivalence between the inverse radiation and the path integral 
pictures for this laser-acceleration geometry can be shown to hold in the nonrelativistic limit as well [11].

Figure 5 shows the energy gain angular dependence of the laser beam with a highreflector boundary at $45^{\circ}$. It shows that for $\gamma>>1$ the angular dependence is almost symmetric and very narrow. The small asymmetry would vanish in the highly relativistic limit and becomes substantial for non-relativistic situations.

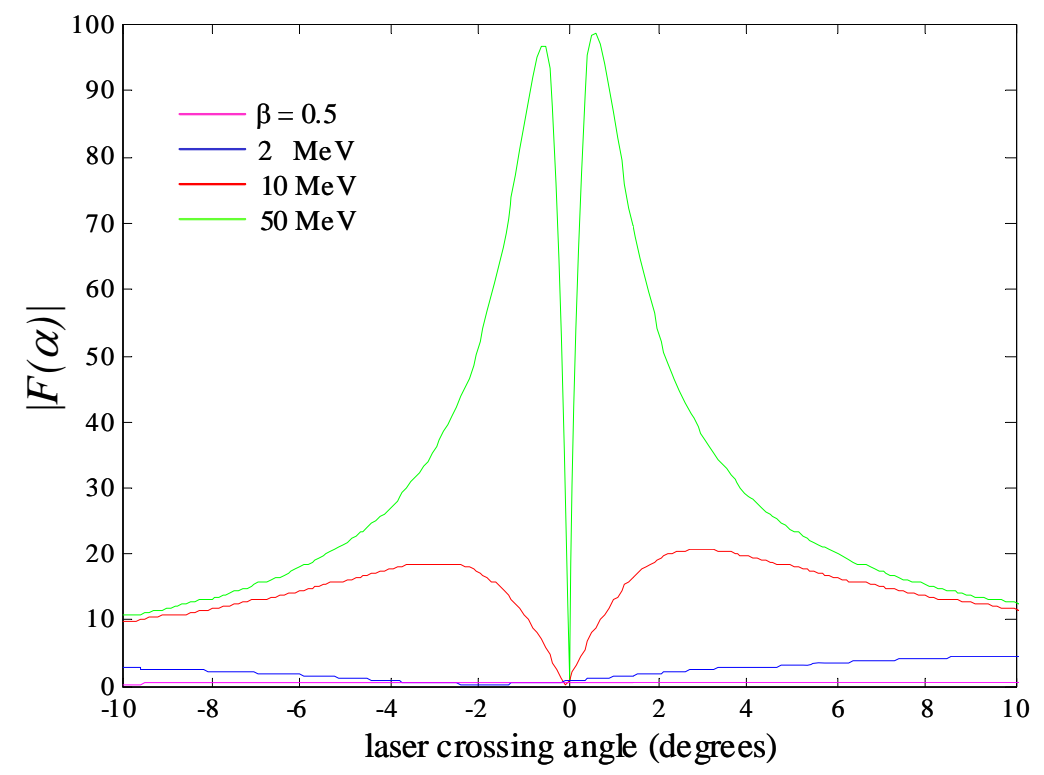

FIGURE 5. Angular dependence of the energy gain at different initial particle energies. The high reflector surface is oriented at $45^{\circ}$

\section{Case (b): The absorptive boundary}

This case is interesting from an experimental point of view because it represents the one instance where the inverse-radiation picture as formulated in equation 1 may not apply. In this situation energy dissipation of the laser from the medium is taking place. In the limit where the boundary is an ideal black absorber there is no reflected laser electromagnetic wave component and a naïve application of the inverse-radiation overlap formula would predict no energy gain for the relativistic particle. The path integral picture makes no assumption about the boundary type other than its ability to stop or deflect the laser, and in the relativistic limit predicts an energy gain similar to that from a reflective boundary. Therefore it will be interesting to compare the observed energy gain for a reflective and black absorbing boundary under similar electron beam conditions to explore the validity of the path integral method with a boundary that absorbs energy from the laser beam. 


\section{Case (c): The transparent boundary}

From a staging perspective this case is probably the most interesting. Under certain boundary configurations we expect a phase-reset of the laser beam and hence an effective doubling of the slippage distance due to addition of the energy gain from the downstream space. It is expected that the total energy gain should show an interference effect between the interactions in upstream and downstream regions that depends on the optical delay caused by the dielectric plate. In the experiment the boundary will be oriented near Brewster's angle with respect to the laser beam, such that there is no significant reflected laser beam component. Figure 6 illustrates the geometry of the transparent boundary.

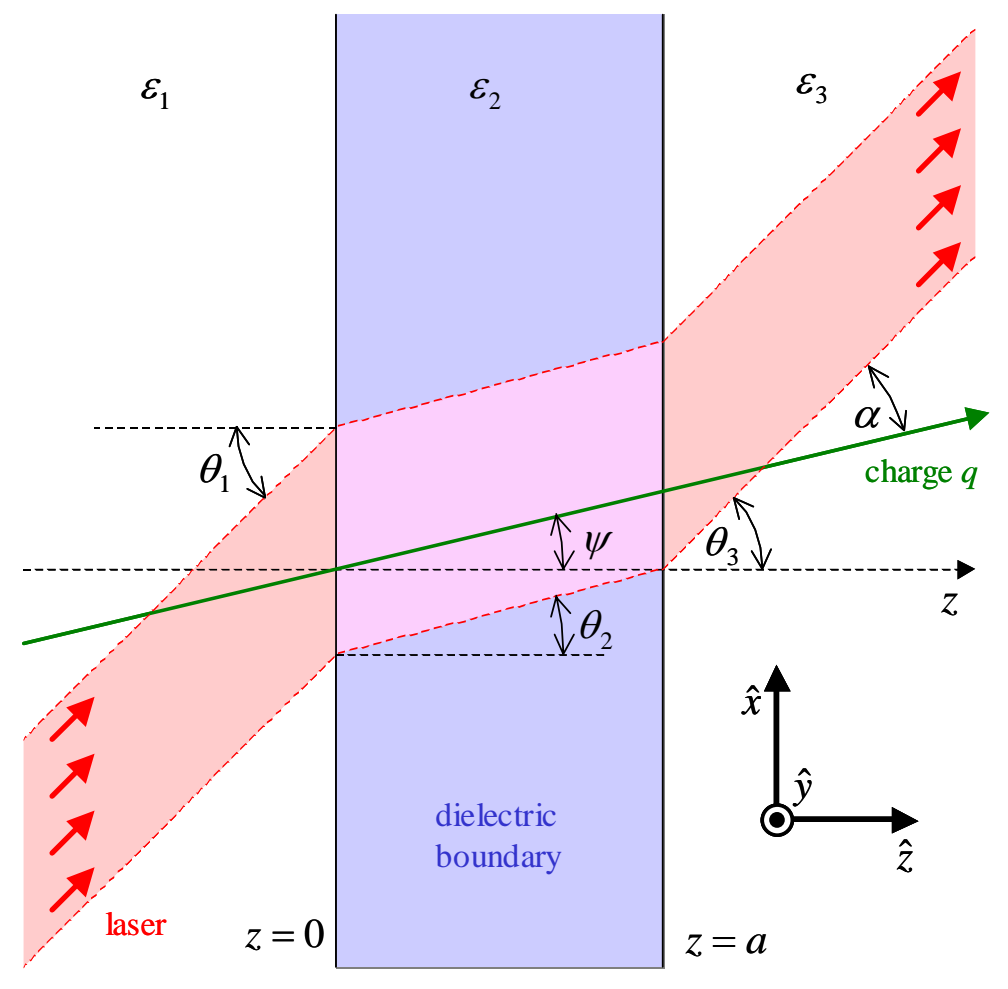

FIGURE 6. The laser-electron beam interaction in the presence of three different dielectric layers. In the experiment the vacuum regions have dielectric constants

The boundary is very thin and therefore its contribution the particle's energy gain is neglected. The energy gain from the upstream and downstream vacuum spaces have the same form as equation 2, but differ by an optical phase delay factor caused by the boundary

where $a$ is its thickness, has the usual definition and and are the laser crossing angles in the medium and the vacuum space respectively. It is 
assumed that the laser beam is fully polarized in the $x z$ plane, such that there is no spolarization component incident on the dielectric interfaces and hence no significant reflection components from these, since they are assumed to be at near-Brewster angle. By either the inverse-radiation or the electric field path integral picture the total energy gain is found to have the form [12].

Figure 7 illustrates the expected maximum energy gain for laser-driven particle acceleration in the presence of a reflective boundary and in the presence of a transparent boundary as a function of the optical phase delay. At optical phase delay values corresponding to the total energy gain is zero and for delay values corresponding to the energy gain in the presence of the transparent boundary has twice the value for that in the presence of a reflective boundary.

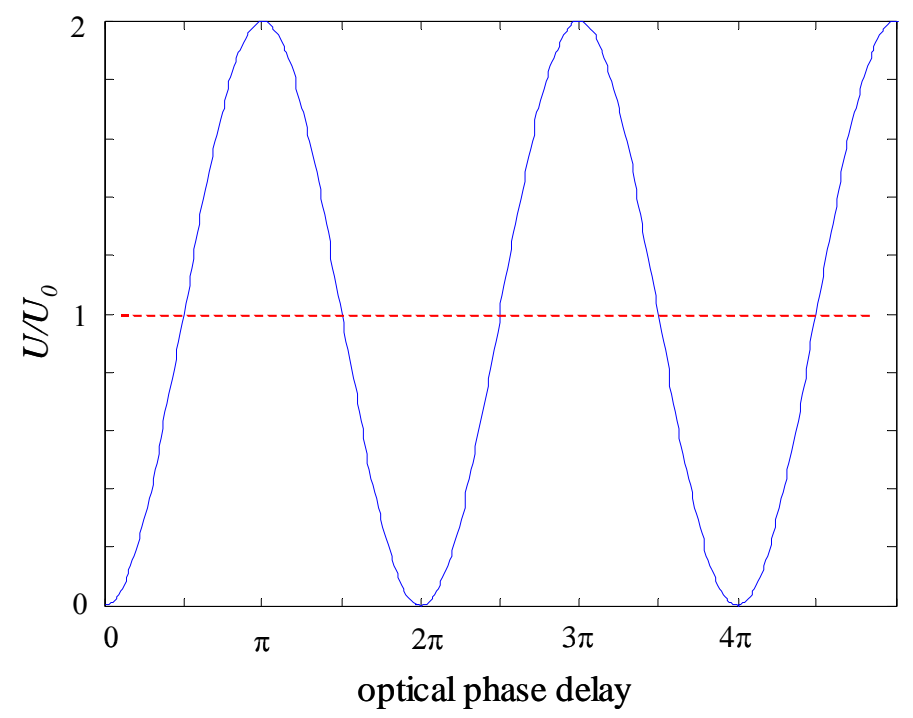

FIGURE 7. Comparison between the energy gain from a reflective boundary (dashed line) and the energy gain from a transparent boundary (solid line) as a function of the optical phase delay

\section{PROPOSED SETUP FOR THE PLANAR BOUNDARY LASER- ACCELERATION EXPERIMENTS}

The design will include a series of minor changes to the original setup employed for the proof-of-principle experiment [10]:

- The ability to rotate the boundary to arbitrary angles. For the measurements with the transparent boundary the orientation will be set to near Brewster's angle. 
- A reflected laser beam diagnostic that records the reflected laser beam profile on a shot-to-shot basis to allow for correlation with the individual laseracceleration events.

- An optical phase-sensitive transmitted laser beam diagnostic to measure the optical delay from the transparent boundaries

Similar to the proof-of-principle experiment, this set of measurements will include only one laser-accelerator stage and therefore the laser-electron interaction signature will be an energy modulation. The electron beam will consist of $1 \mathrm{psec}, \sim 10 \mathrm{pC}$ electron bunches with an energy of $60 \mathrm{MeV}$ and an energy spread of $\sim 20 \mathrm{keV}$. A 2 psec, $\sim 1 / 2 \mathrm{~mJ} /$ pulse, $\lambda=800 \mathrm{~nm}$ laser beam focused to a waist of $\sim 100 \mu \mathrm{m}$ will be employed for the laser-electron interaction. The boundary will consist of a very thin film that will be displaced in a tape-player fashion for each new event, presenting an unexposed surface area to the laser and the electron beam. A schematic of the experiment showing the layout for laser-acceleration experiments in the upstream space of the boundary is presented in Figure 8. Similar to the proof-of-principle experiment an energy spectrometer placed downstream will be used to record the changes of the energy spectrum resulting from the laser-electron interaction.

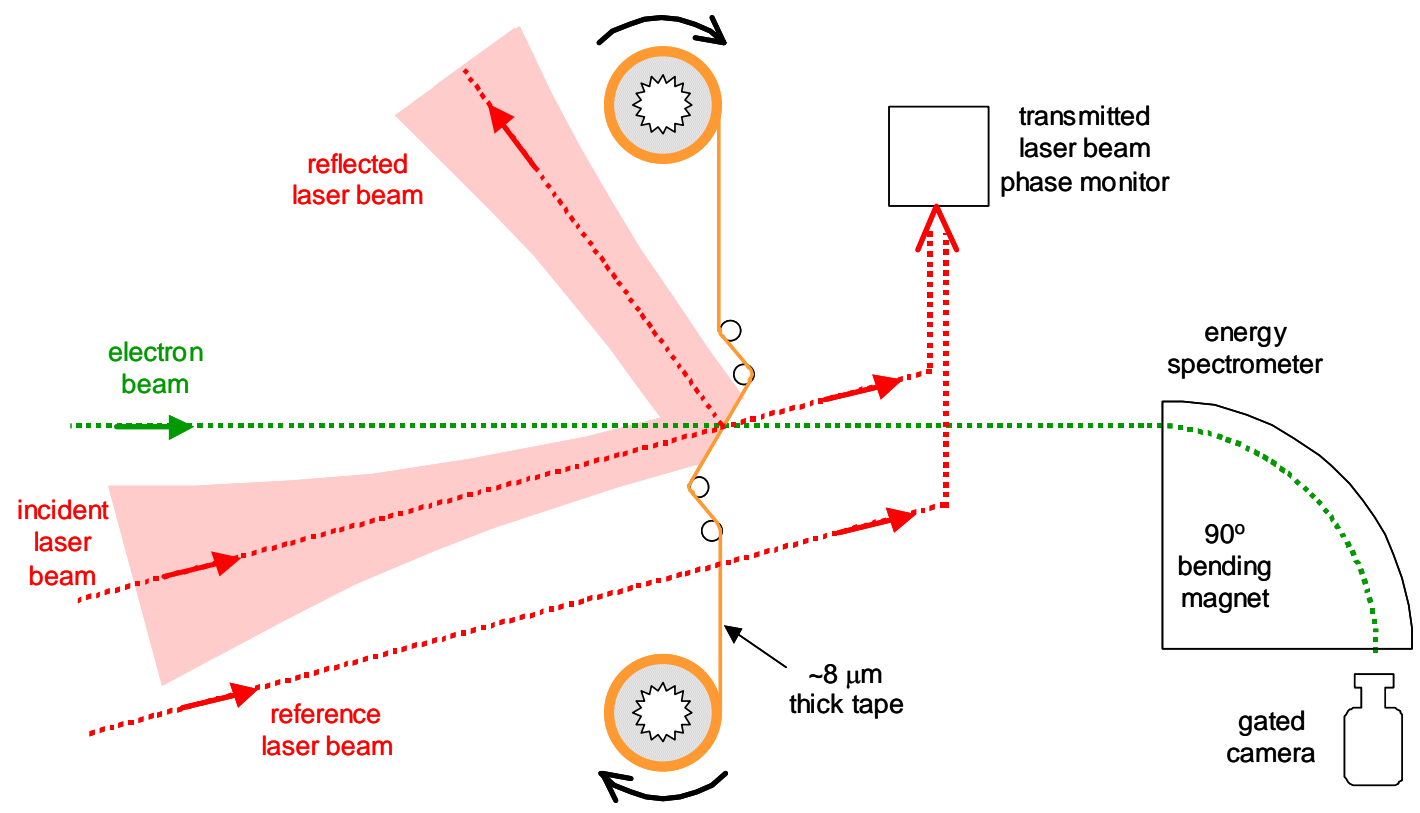

FIGURE 8: layout of the experiment for laser-electron interaction in the upstream space. The reference laser beam will not traverse the film and will be interfered with the transmitted laser beam

The boundaries to be tested will consist of different types of few-micron thick polymer films coated or uncoated. Although the electron beam will traverse the film no significant energy broadening and energy loss is expected to occur, as was observed in the original proof-of-principle experiment with the gold-coated film [13]. The laser beam will be oriented at laser crossing angles that optimize the interaction in 
the vacuum, and therefore the residual inverse-Cerenkov interaction inside the film will be negligible and will not interfere with the physics that is to be explored. Table 2 lists the specific films that are to be tested as boundaries.

TABLE 2. Description of the boundaries to be tested

\begin{tabular}{lll}
\hline Film type & film thickness & boundary \\
\hline Au-coated Kapton & $8 \mu \mathrm{m}$ Kapton, $1 \mu \mathrm{m} \mathrm{Au}$ & reflective \\
Al-coated Kapton & $8 \mu \mathrm{m}$ Kapton, $1 \mu \mathrm{m} \mathrm{Al}$ & reflective \\
clear Kapton & $8 \mu \mathrm{m}$ Kapton & transparent \\
clear polyester & $6 \mu \mathrm{m}$ polyester & transparent \\
black Kapton & 1 mil thick & absorptive \\
audio tape & $13 \mu \mathrm{m}$, different layers & absorptive \\
tape cleaner & $\sim 1 \mathrm{mil} \mathrm{fiber}$ & rough surface \\
\hline
\end{tabular}

The last boundary in table 1 , the tape cleaner, is the only non-planar boundary to be tested in this set of measurements. It has a rough surface with features larger than the wavelength of the laser and hence will produce a complicated reflected laser and TR pattern whose overlap is not obvious. Table 3 lists the important experiment parameters and the expected maximum energy modulation values for the different boundaries

TABLE 3. Relevant experiment parameters and expected energy modulation values

\begin{tabular}{lll}
\hline Parameter & value \\
\hline electron beam parameters & $60 \mathrm{MeV}(\gamma \sim 120), \tau \sim 1 \mathrm{psec}$ \\
laser beam parameters & $1 / 2 \mathrm{~mJ} / \mathrm{pulse}, \lambda=800 \mathrm{~nm}, \tau \sim 2 \mathrm{psec}, \omega_{0} \sim 100 \mu \mathrm{m}$ \\
corresponding peak field & $E_{0} \sim 4 \mathrm{GV} / \mathrm{m}$ \\
optimum laser crossing angle & $\alpha=1 / \gamma \sim 8.3 \mathrm{mrad}, Z_{\text {slippage }} \sim 5.8 \mathrm{~mm}$ \\
upstream energy gain & $\Delta U_{\max } \sim 60 \mathrm{keV}$ & \\
\hline Maximum energy gain & upstream config. & downstream config \\
\hline Reflective boundary & $\Delta U_{\max }$ & $\Delta U_{\max }$ \\
Absorptive boundary & $\Delta U_{\max }$ & 0 \\
Transparent boundary & $0<\Delta U<2 \Delta U_{\max }$ & 0 \\
Rough surface & $\Delta U_{\max }$ & 0 \\
\hline
\end{tabular}

From a practical perspective the transparent boundary case is most interesting since it will be the first primitive example of staged interaction, where the boundary acts as an optical phase-reset device. In principle, further addition of a handful of thin boundaries could extend the interaction length, effectively providing for a rudimentary few-period "structure loaded" vacuum structure that proves the concept of upcoming experiments with more complex guided wave structures.

\section{TWO-STAGE BUNCHER-ACCELERATOR EXPERIMENTS}

After the completion of the single-boundary experiments our next major objective is to observe microbunching at near-infrared wavelengths and subsequently to carry out a two-staged, net laser acceleration experiment comparable to the staged IFEL acceleration experiment at $10 \mu \mathrm{m}$ by Kimura et al [2] but at near-IR wavelengths. The 
first stage will use an IFEL that acts as an optical buncher. The IFEL will be followed by a chicane to turn the energy modulation from the IFEL interaction into a density modulation. The electrons then enter the accelerator and then to the spectrometer for analysis. The expected microbunching has approximately 4 times the electron density at the peak phase versus $\pi$ out of phase with a FWHM width of about $\lambda / 6$. Simulations of the experiment show most of the laser power should go to the accelerator structure to maximize the net acceleration (Figure 9). Simulation of the entire experiment shows we can expect to see $\sim 17 \mathrm{keV}$ of net acceleration from the second accelerator.
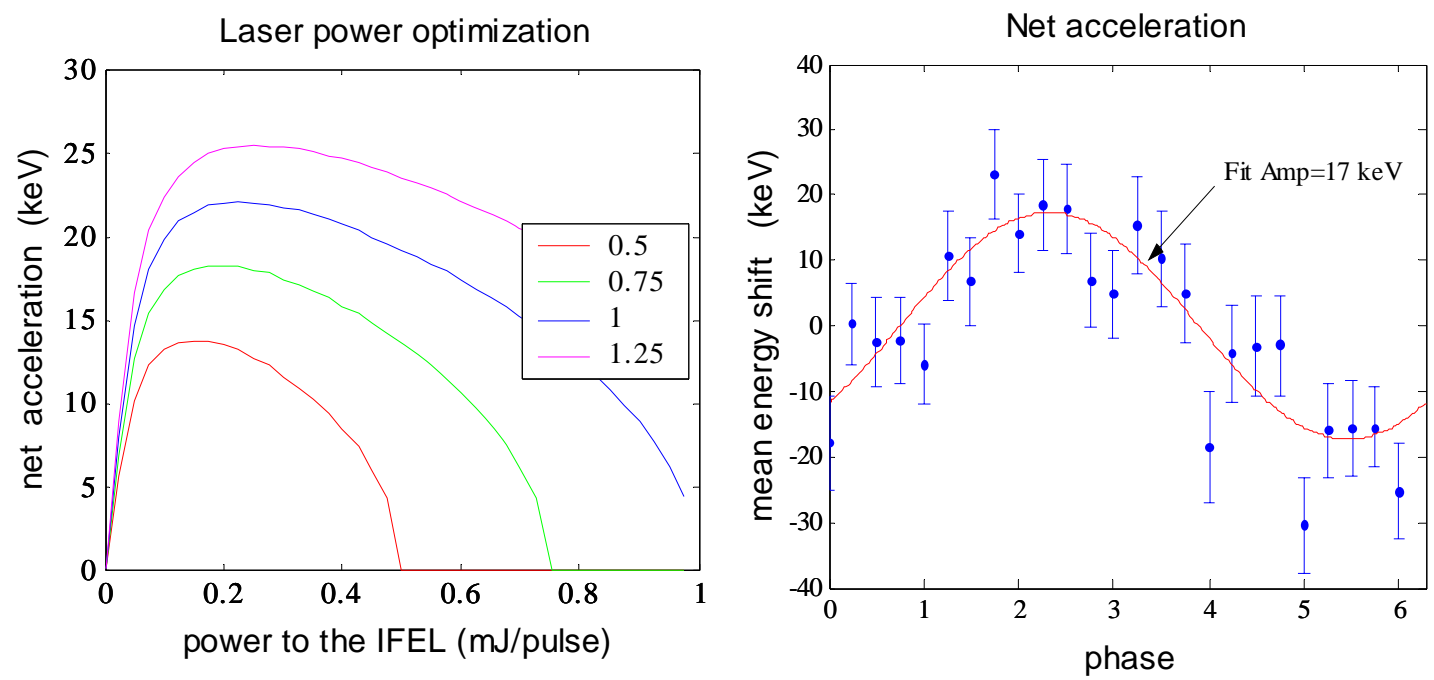

FIGURE 9. a) Laser power splitting optimization. Experiment will likely run with $1 \mathrm{~mJ} / \mathrm{pulse}$ available for the experiment implying use of a 30/70 splitter. b) Scan of buncher-accelerator phase shows change in mean energy of the beam, giving net acceleration of $\sim 17 \mathrm{keV}$ from the single boundary accelerator. Simulations of the experiment show most of the laser power should go to the accelerator structure to maximize the net acceleration (Figure 10). Simulation of the entire experiment shows we can expect to see $\sim 17 \mathrm{keV}$ of net acceleration from the second accelerator.

\section{PHOTONIC BANDGAP ACCELERATOR STRUCTURES}

An appealing aspect of photonic bandgap accelerator structures is that these show potential for relatively large structure impedances and that fabrication technique for these already exist. Photonic bandgap fiber accelerator structures have been proposed and their ability to sustain accelerating modes is being investigated extensively both by computational modeling and by bench top measurements. Beam experiments employing short sections of photonic bandgap fibers as laser-accelerators are in the design phase. The proposed experimental setup is shown in Figure 10. The electron beam enters the fiber through a thin pellicle and exits through a second thin pellicle that allows monitoring of the transmitted laser light. The focusing of the incoming electron beam and measurement of the energy modulation would use the same apparatus described earlier. 


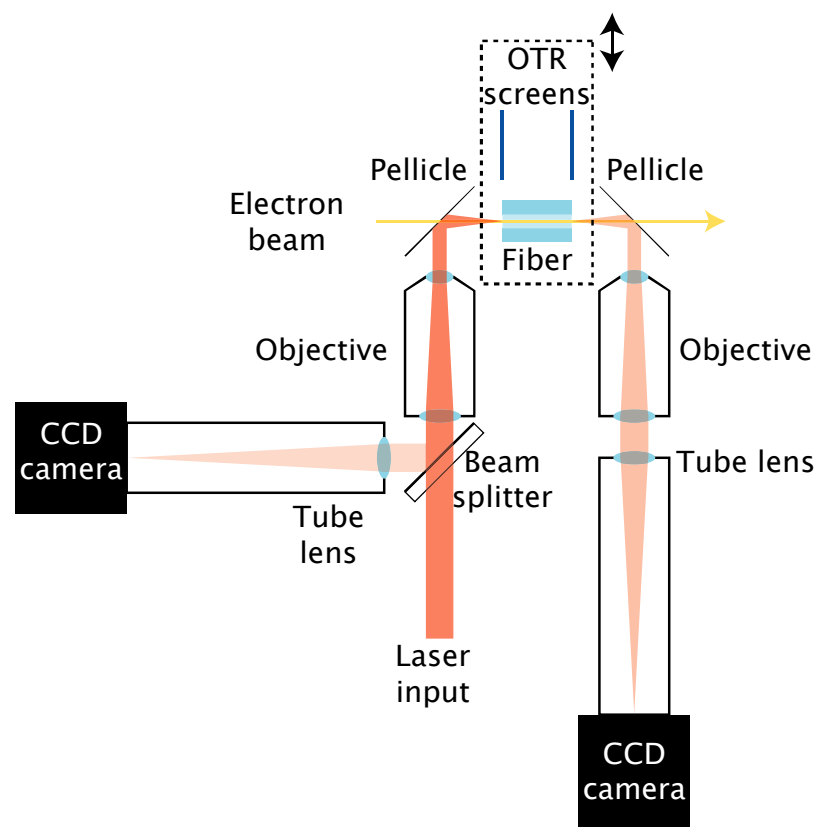

FIGURE 10. The proposed setup for a photonic band gap fiber accelerator experiment.

In addition to the hollow-core fibers a 3-dimensional photonic bandgap structure using a woodpile configuration is being studied [15,16]. Figure 11 shows the geometry of the woodpile photonic bandgap structure. The waveguide confines the speed-oflight mode in both transverse dimensions, and has an extraordinarily high characteristic impedance for an optical structure. At an expected $410 \Omega$, the impedance is high enough that with $10 \mathrm{~kW}$ of peak power in the waveguide, easily attainable from commercially available fiber lasers, the gradient exceeds $1 \mathrm{GeV} / \mathrm{m}$. In addition to the continuation of theoretical modeling of the fields in the structure a significant effort to determine the laser-damage threshold of silicon is in progress.
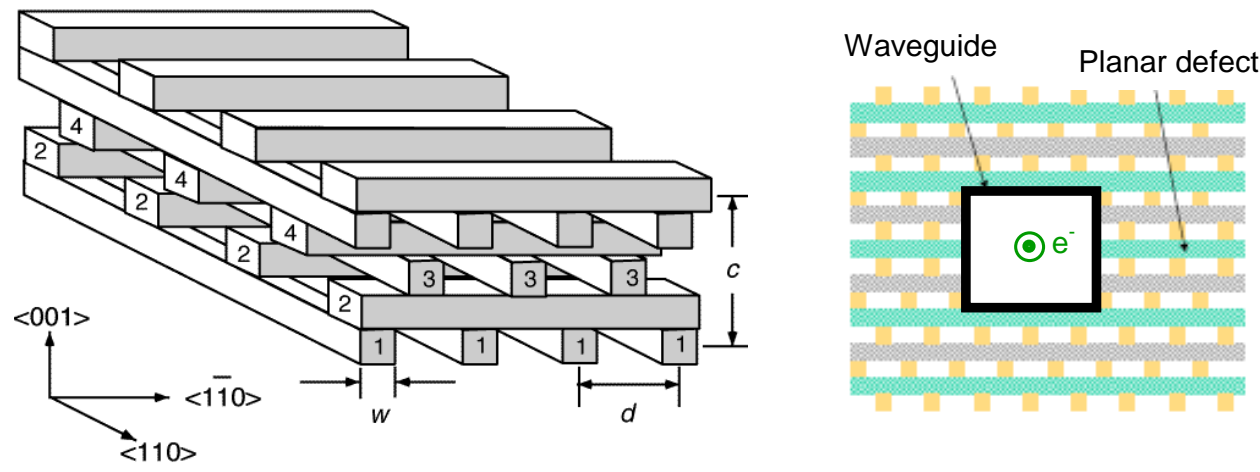

FIGURE 11. LHS) A three-dimensional woodpile lattice without a defect that has an omni-directional bandgap. RHS) End-on view showing the rectangular waveguide and planar defect introduced to create an accelerating structure. 


\section{CONCLUSIONS}

The near-term future experiments described in this article are a natural extension of the initial proof-of-principle experiment. They are designed to test fundamental laseraccelerator physics concepts and also present a first opportunity to explore anticipated challenges such as micro-alignment, beam transport and laser coupling. In conclusion, they constitute an important starting point for the long-term objective of extended laser-driven particle accelerator structure development.

\section{ACKNOWLEDGMENTS}

We would like to thank Mike Hennessy for his valuable assistance in setting up the hardware for the experiment at the SCA-FEL facility. We would also like to thank the personnel at that facility for their assistance in running the accelerator. This work is supported by Department of Energy contracts DE-AC02-76SF00515 and DE-FG0203ER41276.

\section{REFERENCES}

1. T. Plettner, R.L. Byer, E. Colby, B. Cowan, C.M.S. Sears, J. E. Spencer, R.H. Siemann, "Visible-laser acceleration of relativistic electrons in a semi-infinite vacuum”, Phys. Rev. Lett. 95, 134801 (2005)

2. W. D. Kimura, et al., "First Staging of Two Laser Accelerators", Phys. Rev. Lett. 86, p. 4041-4043 (2001).

3. X. Eddie Lin, "Photonic band gap fiber accelerator”, Phys. Rev. ST Accel. Beams 4051301 (2001)

4. B. Cowan, "Photonic crystal laser-driven accelerator structures” SLAC-PUB-11192 (2005)

5. J.A. Edinghofer, R.H. Pantell, "Energy Exchange between free electrons and light in vacuum”, J. Appl. Phys. 506120 (1979)

6. M. Xie, “A Fundamental Theorem on Particle Acceleration”, Proceedings of the 2003 Particle Accelerator Conference (2003)

7. Z. Huang, G. Stupakov and M. Zolotorev , "Calculation and Optimization of Laser Acceleration in Vacuum”, Phys. Rev. Special Topics - Accelerators and Beams,Vol. 7, 011302 (2004)

8. See for example Jackson, Classical Electrodynamics, $2^{\text {nd }}$ edition, chapter 6, p 236

9. Hung-chi Lihn, “Stimulated Coherent Transition Radiation”, SLAC-R-480, 1996

10. M.L. Ter-Mikaelian, "High-Energy Electromagnetic Processes in Condensed Media” Interscience Tracts on Physics and Astronomy, John Wiley\&Sons, Inc.

11. T. Plettner, "Analysis of Laser-Driven Particle Acceleration from Planar Infinite Conductive Boundaries”, SLAC-PUB-11637 (2006)

12. T Plettner, “Analysis of Laser-Driven Particle Acceleration from Planar Transparent Boundaries”, SLAC-PUB-11800 (2006)

13. T. Plettner, R.L. Byer, E. Colby, B. Cowan, C.M.S. Sears, J. E. Spencer, R.H. Siemann, "Proof-ofprinciple experiment for laser-driven acceleration of relativistic electrons in a semi-infinite vacuum", Phys. Rev. ST Accel. Beams 8, 121301 (2005)

14. C.M.S. Sears, E. Colby, B. Cowan, J. Spencer, R.H. Siemann, R.L. Byer, T. Plettner, "High Harmonic Inverse Free Electron Laser Interaction at 800 nm”, Phys. Rev. Lett. 95, 194801 (2005)

15. B. Cowan, M. Javanmard and N. Wu, "Photonic Crystal Laser-Driven Accelerator Structures", SLACPUB-10590 (2004)

16. B. Cowan, "Photonic Crystal Laser-Driven Accelerator Structures”, SLAC-PUB-11192 (2005) 